\title{
JOGOS ELETRÔNICOS DE MOVIMENTO E EDUCAÇÃO FÍSICA: UMA REVISÃO SISTEMÁTICA
}

\author{
Letícia de Castro Silva Monteiro \\ Universidade Federal de Goiás, Goiânia, Goiás, Brasil \\ Fátima Solange Castillo Velásquez \\ Universidade Federal de Goiás, Goiânia, Goiás, Brasil \\ Ana Paula Salles da Silva \\ Universidade Federal de Goiás, Goiânia, Goiás, Brasil
}

\begin{abstract}
Resumo
O objetivo desta pesquisa é realizar uma revisão sistemática dos artigos científicos produzidos sobre Jogos Eletrônicos de Movimento, apontando seus usos para o campo da Educação Física. As pesquisas encontradas indicam o uso dos Jogos Eletrônicos de Movimento como instrumento de intervenção no campo da reabilitação, da promoção da saúde e da prática pedagógica. Aparece também enquanto conteúdo pedagógico na Educação Física Escolar. Os estudos realizados não apontam os sentidos atribuídos pelos usuários para além daqueles relacionados à saúde.
\end{abstract}

Palavras chaves: Jogo Eletrônico. Educação Física. Educação. Saúde.

\section{Introdução}

Os Jogos Eletrônicos surgem na década de 1970 como uma inovação tecnológica bem sucedida no mercado de entretenimento, mas atualmente as vendas de Jogos Eletrônicos são mais expressivas do que quando lançados, fazendo com que o mercado dos jogos superasse o faturamento da indústria cinematográfica nos últimos anos (AMARAL; PAULA, 2007). Parte importante nos lucros dos grandes desenvolvedores de consoles caseiros e Jogos Eletrônicos na última década refere-se aos consoles para Jogos Eletrônicos de Movimento (JEMs). Os JEMs caracterizam-se por valorizar a experiência com o movimento durante a experiência, sendo uma das possibilidades existentes de Jogo Eletrônico.

Ao contrário do que se imagina e do que era veiculado pela mídia, os JEMs lançados na década passada, segundo Silva (2012), não eram novidades no mercado dos Jogos Eletrônicos, a diferença é que não haviam obtido o mesmo sucesso nas vendas.

Ainda segundo essa autora, este fracasso inicial estava associado ao custo elevado dos JEMs e dos acessórios necessários para jogá-los, e também a baixa eficiência dos JEMs e dos acessórios em termos de jogabilidade. Um bom exemplo foi o lançamento, em 1982, do acessório "The JoyBoard", da empresa Amiga, para ser acoplado ao console Atari 2600. O "The JoyBoard", salvo as devidas diferenças tecnológicas, apresentava a mesma intencionalidade do "Balance Board", acessório desenvolvido e apresentado como novidade pela empresa Nintendo em 2006.

A melhoria no desenvolvimento tecnológico dos JEMs e seus acessórios, a baixa nos custos e a sua visibilidade despertaram o interesse da população de diferentes faixas etárias, em especial, pelo fomento à interação social e à atividade física. Em relação à atividade física é importante destacar esta novidade, já que no campo científico a prática do Jogo Eletrônico 
induz ao sobrepeso e a obesidade pelo baixo gasto energético durante a prática (SILVA et al., 2010).

A relação dos Jogos Eletrônicos com o movimento e até mesmo com o conteúdo esportivo, abriram novas perspectivas para a pesquisa científica na Educação Física na relação com a tecnologia. Neste sentido, nos interessa saber neste trabalho quais foram os caminhos trilhados na compreensão dos sentidos e usos dos JEMs para este campo de atuação.

Esta problemática justifica-se pelas possibilidades de identificar similaridades em torno das pesquisas desenvolvidas, o que contribui para apresentar tendências em relação a este fenômeno, mas também na identificação de lacunas, indicando novas demandas de estudos para o campo da Educação Física.

\section{Metodologia}

Foi realizada uma revisão sistemática da literatura através do Google Acadêmico, em maio de 2015, utilizando como descritores os termos "exergames" e "wii", ambos em virtude da sua recorrência na produção acadêmica. A pesquisa foi limitada aos artigos produzidos em língua portuguesa, com a intenção de identificar a produção nacional a cerca do tema. Para realização desta pesquisa foram considerados apenas artigos científicos publicados em revistas, sendo encontrados 32 referências que correspondiam a artigos científicos com o termo "exergames" e 17 referências que correspondiam ao termo "wii".

Para o processo de análise foram estabelecidos os seguintes critérios de exclusão: artigos de revisão sistemática; artigos que não abordavam os JEMs como tema principal da pesquisa, artigos que se repetiam entre os termos de busca; artigos que apresentavam uso da tecnologia de JEM para outros fins; e artigos não disponíveis para download. Após a verificação inicial dos artigos foram selecionados para análise 24 artigos. A partir do processo da análise os textos foram organizados em 04 eixos-temáticos, quais sejam: JEM e Reabilitação; JEM e Atividade Física; Sobre a experiência com o JEM; e JEM e Escola. Os eixos-temáticos foram organizados a partir dos conteúdos desenvolvidos nos artigos.

\section{JEM e reabilitação}

Os artigos reunidos neste eixo-temático apontam o uso das pesquisas como instrumento de reabilitação. Sobre este aspecto Sposito e colaboradores (2013), Mussato, Brandalize e Brandalize (2012), Treml e colaboradores (2013) apontam que esta tecnologia interativa é capaz de imergir, integrar e envolver o usuário ao utilizar dispositivos multissensoriais, a partir de um movimento tridimensional. Destaca-se ainda que o uso dos JEMs evita a monotonia típica do tratamento fisioterapêutico tradicional. (SPOSITO et al., 2013).

O console Nintendo Wii é o mais utilizado em processos de reabilitação, treinamento neuromuscular proprioceptivo e estudos sobre parâmetros fisiológicos gerados pelo tipo de atividade exercida durante o jogo. (SPOSITO et al., 2013; TREML et al., 2013.)

Em relação à reabilitação os artigos apresentam possibilidades positivas de intervenção no caso de sequelas de patologias e também de deficiências.

O estudo realizado por Grande, Galvão e Gondim, L. (2011) verificou o ganho de amplitude de movimento ativo das articulações do membro superior esquerdo, em um paciente com lesão alta de nervos periféricos. Utilizaram o software Nintendo Wii como instrumento terapêutico com três jogos diferentes, os quais utilizaram movimentos de duas ou mais articulações analisadas. O paciente teve ganho de amplitude significativo nas articulações analisadas, e após a intervenção o mesmo voltou a realizar atividades diárias com independência e retomou algumas atividades esportivas. 
Schiavinato e colaboradores (2009) realizaram sua intervenção em um paciente com 23 anos diagnosticado com Ataxia Cerebelar Precoce. Utilizou-se o JEM Wii Fit durante nove sessões de 30 minutos cada. Os pesquisadores encontraram resultados positivos, como melhora no equilíbrio, na execução de Atividades de Vida Diária e Atividades de Vida Prática, aumentando o nível de independência do paciente.

Zeigelboim e colaboradores (2013) utilizaram o JEM através do Wii Fit em adultos com ataxia espinocerebelar, com uma amostra de quatro pessoas com faixa etária superior a 30 anos, das quais três obtiveram melhoras no equilíbrio e na coordenação motora, e apenas uma se manteve estável nas mesmas habilidades.

No artigo de Periard, Elias Filho e Augusto-Silva (2013) é feito um estudo de caso com um paciente idoso com sequela de Acidente Vascular Encefálico em fase crônica, objetivando a melhora funcional do seu membro superior parético. Em relação ao seu membro superior afetado, o paciente hemiparético teve melhoras na funcionalidade e aumento da amplitude de movimento. O que sugere que a utilização do Nintendo Wii como instrumento de reabilitação é eficaz na melhora da funcionalidade do membro afetado.

$\mathrm{Na}$ reabilitação de um paciente com Charcot Marie Tooth (conhecida como Atrofia Fibular Muscular), Franco e colaboradores (2013) utilizaram o Wii Fit, obtendo resultados positivos na percepção visual, mobilidade funcional e ajuste postural do indivíduo, e pontuou ainda que o dispositivo possui um custo $\mathrm{x}$ benefício muito favorável, sendo capaz de trabalhar disfunções motoras relacionadas ao equilíbrio de maneira eficaz, além de se adaptar às limitações do paciente.

Avaliando a eficácia imediata na utilização do Nintendo Wii como intervenção fisioterapêutica em pacientes idosos com Parkinson, Silva e colaboradores (2011), notaram em seu experimento uma melhora, ainda que não significativa, da agilidade e do equilíbrio. A resposta para este resultado não significativo da utilização do Wii como ferramenta pode ser reflexo de ter se realizado apenas uma sessão.

Frade e colaboradores (2014) realizaram uma pesquisa utilizando o Software Wii Fit com um público jovem com deficiência visual, classificados com baixa visão, sua intervenção fora dividida em duas etapas, uma de nível fácil e outra de nível difícil. Participaram da primeira etapa dez jovens, e da segunda etapa apenas quatro, um por ter feito a maior pontuação do jogo na primeira etapa, e os outros três por demonstrarem interesse. Ambos os grupos apresentaram melhoras significativas no equilíbrio.

Ao contrário de Silva e colaboradores (2011), citado mais acima, Frade e colaboradores (2014) verificaram com a utilização do Nintendo Wii Fit associado à plataforma Balance Board melhora significativa no equilíbrio de todos os participantes. $\mathrm{O}$ que indica que para a reabilitação de pessoas com deficiência visual o uso de JEMs apresenta resultados promissores, no entanto, no caso de doença de Parkinson há necessidade de maiores estudos para avaliar seu potencial de reabilitação.

Silva e Iwabe-Marchese (2015) realizaram sua intervenção utilizando o software Wii Fit Plus associado à Balance Board, sua amostra contou com uma criança com Paralisia Cerebral Atáxica, com 40 sessões de 30 minutos cada. Os jogos utilizados tinham como foco o equilíbrio e o deslocamento de peso de maneira estática, trabalhavam de maneira secundária a marcha. A criança apresentou um desenvolvimento significativo quanto ao equilíbrio, e nem tanto quanto a marcha.

Na pesquisa de Pavão e colaboradores (2014) foi realizada uma intervenção de 12 sessões em uma criança de sete anos que apresenta Paralisia Cerebral hemiplégica com comprometimento funcional leve. Eles utilizaram o software Xbox 360 com Kinect, os dois jogos utilizados exigiam a movimentação do jogador com movimentos dos membros superiores e inferiores na diagonal, treino de destreza para atingir alvos específicos, movimentos de grande amplitude, requerendo a utilização de tronco para gerar transferências 
de peso, agachamentos e saltos. Ao final da intervenção a criança obteve ganhos no desempenho motor e equilíbrio funcional, também observaram ganhos na motricidade fina da criança.

Através de um estudo de caso, Medeiros e colaboradores (2013), avaliaram o uso do Xbox 360 com Kinect em um caso que o paciente com lesão medular, com 63 anos de idade, apresentando quadro sindrômico de tetraparesia espástica, com alteração de equilíbrio e marcha, dependente de dispositivo auxiliar (andador/barras paralelas). A escolha da plataforma foi devido ao paciente não conseguir se manter de pé sem o auxílio de dispositivo, não conseguindo manipular o controle do Nintendo Wii. O paciente participou de 15 sessões, com jogos de corredeira (Kinect Adventures). O paciente demonstrou melhoras físicas e cognitivas, adquiriu novamente confiança em si mesmo, redução dos medos de quedas, equilíbrio ortostático, destreza, condicionamento físico aeróbico, e resistência muscular. $\mathrm{O}$ feedback visual imediato foi um dos fatores mais importantes para seu desenvolvimento, fazendo com que o indivíduo se demonstrasse mais concentrado, fazendo esforço físico, e obtendo prazer com o entretenimento.

As pesquisas analisadas tratam de patologias e deficiências diferentes que comprometem a condição física, sendo que destas sete se configuram como estudos de caso, ou seja, com apenas um paciente submetido à intervenção com JEMs sendo avaliado. A melhora do equilíbrio através das intervenções realizadas com JEMs ficou evidente em oito das dez pesquisas, incluindo as três pesquisas com mais pacientes sendo avaliados, caracterizando o uso JEMs como uma ferramenta promissora na intervenção de patologias e deficiências que afetam o equilíbrio. A melhora ainda que não significativa da agilidade verificada no estudo com pacientes com parkinson também apresenta indícios promissores na reabilitação, em virtude de apresentar uma amostra de nove pessoas e pela análise ter sido realizada após uma única sessão de intervenção, enquanto que os outros estudos realizaram no mínimo nove sessões. Os outros ganhos conquistados dependem em nossa análise de novas pesquisas para serem validados, visto que apresentam recorrência em apenas um ou dois estudos.

\section{JEM e atividade física}

Neste eixto-temático foram reunidas as pesquisas experimentais, que organizaram sessões de intervenção com os JEMs para análise de respostas fisiológicas em relação a atividade física exercida durante a prática. Os JEMs nestas pesquisas aparecem como aliados na promoção da saúde em virtude do dispêndio energético, pelas respostas cardiovasculares positivas e por ganhos na coordenação motora, equilíbrio e na cognição.

Três pesquisas apontam dados positivos sobre o dispêndio energético durante a prática dos JEMs. Santo e colaboradores (2014) avaliaram o dispêndio energético de 20 crianças, com idade entre oito e dez anos, durante a prática de JEMs. As crianças foram submetidas a três sessões de JEM de dez minutos cada com intervalo de cinco minutos entre elas. Os resultados indicaram níveis de intensidade de moderado a vigoroso. Podendo esta prática, segundo os autores, ser indicada para as crianças com o objetivo de atender às recomendações de atividade física.

Biscaia, Martins e Alves (2014) analisaram o potencial do JEM MoveFitness, do console Playstation III, como exercício físico voltado à performance aeróbica. Participaram do estudo 30 jovens adultos, entre 18 e 29 anos. O programa do JEM "Fortalecimento de Cardio" proporciona, segundo os autores, atividade adequada por atingir os parâmetros estabelecidos pela American College of Sports Medicine (ACSM) e pela American Heart Association (AHA). 
Os dois artigos tem em comum a identificação do alcance de níveis recomendados de atividade física, o que indica o uso dos JEMs como uma prática doméstica interessante para o combate da obesidade.

Foram realizados também estudos que avaliaram respostas cardiovasculares, Souza et al. (2013) avaliou o JEM de basquetebol do software Wii Resorts, observando que os exercícios realizados no jogo foram de intensidade leve (arremesso sentado) e moderada (arremesso em salto vertical), a partir desta análise eles verificaram que é possível melhorar e manter a aptidão cardiorrespiratória com este jogo, indo ao encontro com as diretrizes do ACSM.

Pirrier-Melo e colaboradores (2013) encontraram resultados semelhantes, mas utilizando o Xbox 360 com o Kinect. Os autores analisaram em uma única sessão a Frequência Cardíaca e Pressão Arterial, durante e após o término de cada jogo respectivamente. Foram utilizados os JEMs do Kinect Sports Vol. 1 e Dance Central 3. Os resultados dessa pesquisa indicam que os JEMs usados foram semelhantes a uma atividade física de intensidade moderada, sendo uma excelente alternativa de lazer em Atividades da Vida Diária. Por sua amostra ser composta apenas por oito jovens adultos, os autores sugerem que para experiências como esta devem ser desenvolvidas com amostras maiores.

Dos artigos relacionados à atividade física, três abordam o impacto da prática com os JEMs por pessoas idosas saudáveis. Segundo Treml e calaboradores (2013), a reabilitação em idosos saudáveis sedentários que utilizam o Nintendo Wii com Balance Board para a melhora do equilíbrio, tem resultados melhores que os métodos tradicionais de reabilitação desenvolvidos com o mesmo público. Estes resultados podem ser justificados segundo os autores devido a interatividade proporcionada com os JEMs. No entanto, Mussato, Brandalize e Brandalize (2012); não encontraram os mesmos resultados com idosos saudáveis ativos, mesmo eles apresentando melhoras significativas nos testes de Apoio Unipodal e Time UP \& $G O$ quando comparado com a pré e pós intervenção com os JEMs, perdem quando comparado com o grupo que utiliza o método tradicional.

No estudo Coutinho e Matos (2014) com pessoas idosas saudáveis outras variáveis foram consideradas na análise da intervenção com o JEM Wii Fit. Os autores avaliaram o equilíbrio, coordenação motora, cognição e desempenho em Atividades da Vida Diária. Os idosos saudáveis apresentaram resultados significativos no equilíbrio, na coordenação motora e na cognição, e apesar do resultado positivo encontrado na realização de Atividades da Vida Diária, este resultado não foi considerado significativo.

Os dados destas pesquisas indicam que os JEMs são atividades físicas que promovem benefícios à saúde para diferentes grupos etários saudáveis, não havendo a princípio contra indicações. Estes dados são relevantes porque contrariam uma das principais críticas sobre o uso dos Jogos Eletrônicos nos estudos com atenção a saúde, antes da popularização dos JEMs, e que apontavam sua prática como um fator de risco para o desenvolvimento do sedentarismo e da obesidade, sobre esta crítica ainda atual conferir Tolocka e colaboradores (2008) e Hardy, Hardel e Bell (2014). Neste contexto, tem-se hoje Jogos Eletrônicos que induzem a imobilidade e JEMs que atendem aos padrões recomendados de intensidade na realização de atividade física, mas é necessário destacar que as consequências resultantes nos dois casos dependem da frequência com que os jogos são praticados pelos jogadores.

\section{Sobre a experiência com o JEM}

Neste eixo-temático foram selecionados os artigos que buscaram realizar uma análise da experiência de usuários com o jogo, mas que não são pesquisas experimentais. Foram identificados três artigos que tratam da experiência com os JEMs. 
Finco e Fraga (2012) realizaram uma netnografia em comunidades virtuais de praticantes do Wii Fit, que é um software de JEM jogado com uma plataforma de equilíbrio. O conteúdo das postagens indicou que o Wii Fit tem uma recepção positiva por parte dos praticantes, incentivando-os à prática regular de exercícios físicos, à escolha por uma alimentação saudável e ao controle de peso. Os pesquisadores destacam ainda que o jogo é um modo pelo qual seus praticantes se atualizam sobre as mais recentes recomendações sobre atividade física e saúde, mas ressalta que estas percepções refletem tendências destas comunidades. Em outro artigo Finco e Fraga (2013) analisaram a inserção dos Jogos Eletrônicos na cultura contemporânea evidenciando os modos de interação com o jogo. Os autores destacam que dos JEMs que já foram desenvolvidos, o software do Wii Fit é o que apresenta o melhor potencial para a construção de um estilo de vida ativo.

Na pesquisa de Rodrigues e colaboradores (2013) foi analisada a percepção de idosas sobre a experiência com aulas de dança e com o JEM Just Dance I do console Wii. Os dados da pesquisa revelam que as idosas reconhecem que ambas as experiências são benéficas, mas relataram a preferência pelas aulas de dança. Os autores ponderam que esta preferência pode estar associada à falta de experiência das idosas com os JEMs e sugerem novas pesquisas acerca desta questão.

As análises realizadas nas três pesquisas sobre a experiência com os JEMs indicam o potencial desta experiência para a promoção da saúde, não diferenciando dos apontamentos realizados nas pesquisas experimentais apresentadas no eixo-temático anterior. Mas, a identificação de comunidades virtuais é um elemento interessante que potencializa a aderência dos usuários aos JEMs e a troca de saberes entre eles, de forma que estas comunidades virtuais são espaços de formação de vínculos interpessoais e de socialização e construção de conhecimento acerca da atividade física e da tecnologia.

Ainda sobre as comunidades virtuais, destaca-se então a falta de pesquisas sobre a experiência de usuários com os JEMs em outros cenários sociais mediados, pois assim como existem comunidades vinculadas a praticantes específicos do JEM Wii Fit que tem como proposta/objetivo a realização de exercícios físicos, existem outras comunidades e outros JEMs, como é o caso dos JEMs Wii Resorts e Kinect Adventures, focados na diversão e interação social. Estudos já apontam os Jogos Eletrônicos como uma prática de lazer/entretenimento (RODRIGUES, 2011; RESENDE \& SILVA, 2012; FERES NETO \& JUBÉ, 2012) e as diferentes características acerca deste modo de apropriação. No entanto, novos estudos são importantes visto que as características do JEMs são diferentes em relação aos Jogos Eletrônicos no modo de intergir, porque inclui os movimentos do corpo, e também nas demandas a serem resolvidas nos jogos que podem modificar os modos e os sentidos de apropriação dos JEMs.

\section{JEM e Escola}

As pesquisas deste eixo-temático revelam como os JEMs vêm sendo pensados no cenário educativo, destaca-se aqui seu uso como ferramenta e conteúdo pedagógico.

Vaghetti, Mustaro e Botelho (2011) investigaram os JEMs em rede e sua utilidade para a Educação Física, explorando o uso para a educação à distância e o e-learning. Foram identificados 57 JEMs de consoles domésticos e 16 novos protótipos de JEMS que foram analisados em relação ao desenvolvimento da aptidão física. O estudo indica que os JEMs podem ser utilizados como instrumento de intervenção à distância na educação básica e na formação inicial em Educação Física.

Na análise crítico-reflexiva de Vaghetti, e colaboradores (2012) sobre a utilização dos JEMs como ferramenta pedagógica, problematiza-se a forma como a Educação Física ainda é ensinada. Segundo os autores o ensino da Educação Física ainda está pautado no esporte de 
rendimento, descartando o lúdico, o que gera uma desmotivação dos alunos quanto ao exercício físico, além de provocar a exclusão dos alunos menos habilidosos. O JEM para Vaghetti e colaboradoes (2012) apresenta-se como uma ferramenta pedagógica inovadora na Educação Física, por ser mais atrativa, lúdica, e inclusiva.

Vieira, K. et al (2014) realizaram uma pesquisa com alunos do sexto ano de uma escola pública municipal da cidade de Rio Grande - RS. Tinham como objetivo saber qual a percepção dos alunos sobre a utilização dos exergames nas aulas de Educação Física. Os pesquisadores utilizaram o console Nintendo Wii como instrumento de intervenção com os JEMs. Os jovens investigados gostaram da experiência com os JEMs e relataram que as intervenções realizadas de modo convencional pelo professor e a que utilizou os JEMs foram bem semelhantes, pois abordavam os mesmos esportes. Em suas análises os autores destacam a importância do JEMs como ferramenta pedagógica e também enquanto conteúdo da Educação Física, já que são uma oportunidade de formar os jovens para a cultura digital.

Baracho, Gripp e Lima (2012) organizaram uma intervenção com oito estudantes, com a intenção de analisar a percepção dos alunos em relação à prática pedagógica realizada com o JEM de Beisebol e a prática pedagógica com o jogo convencional de Beisebol. Os estudantes foram submetidos a dez minutos cada com o JEM de Beisebol e com uma aula de Beisebol convencional. Os resultados indicaram a preferência dos jovens pelo JEM de Beisebol. Os autores apontam a riqueza desta experiência por permitir aos estudantes inserção mediada na cultura digital.

Silva e Braccialli (2014) realizaram uma intervenção pedagógica na aulas de Educação Física de uma turma de quarto ano do ensino fundamental, utilizando o JEM dentro de um projeto de atividade física adaptada. Os resultados indicaram que o uso dos JEMs despertou o interesse dos estudantes e que o estudante com deficiência conseguiu se inserir nas atividades propostas.

Em relação aos estudos percebe-se um investimento de análise maior em relação ao seu uso enquanto ferramenta pedagógica, indicando sua positividade ao possibilitar o acesso à cultura digital. Questionam-se aqui os dados obtidos na pesquisa de Baracho, Grip e Lima (2012), ao dizerem que o uso com os JEMs é preferido pelos estudantes em relação ao ensino dos esportes sem eles, já que a metodologia utilizada com os jovens previu um tempo muito reduzido de intervenção. Os dados de Viera e colaboradores $(2014$, p. 67) são resultantes de um maior tempo de intervenção e apontam que " $70,8 \%$ dos alunos relataram preferência tanto por aulas no pátio da escola quanto com EXGs, 2,1\% relataram sua preferência apenas pelos EXGs e 27,1\% afirmaram que preferem aulas no pátio da escola." Corroborando com estes dados à pesquisa de Silva (2012) desenvolvida com jovens em idade escolar, também aponta que um maior tempo de intervenção implica num interesse dos jogadores pelas duas formas (digital e não-digital) de experenciar as práticas corporais.

$\mathrm{O}$ uso do movimento na experiência digital e como isso provoca novas tensões na relação com as práticas corporais e, por sua vez, nos conteúdos da Educação Física, ainda é pouco explorada nas pesquisas. Armando (2010) propõe três categorias para organizar problematizações acerca dos Jogos Eletrônicos enquanto conteúdo que podem auxiliar em novos estudos sobre JEMs, são elas: a) objeto midiático, com a intenção de interrogar a produção, a distribuição e as comunidades em tornos dos jogos; b) linguagem dos jogos, referindo-se as opções estéticas, as características e as representações presentes; e c) conhecimento para jogar, trata dos problemas postos ao jogador e dos saberes necessários para agir no jogo e suas possibilidades de interação.

Em relação aos dados das pesquisas analisadas, é importante situar que a proposição do uso de Jogos Eletrônicos como ferramenta e conteúdo pedagógico na escola não é novidade na área da educação, sendo a teoria da Mídia-Educação (RIVOLTELLA, 2005; 
FANTIN, 2006) que pauta os modos de inserção das mídias e tecnologias na escola avança ainda em outra dimensão, a expressiva-produtiva.

Nas pesquisas também não foram apontados soluções para enfrentar as dificuldades para a inserção destes JEMs na escola, como ausência de tecnologia apropriada (consoles, acessórios, telas e jogos), espaços físicos apropriados e conhecimento dos professores acerca não só do conteúdo narrativo, mas também dos modos de funcionamento destes jogos. Sendo que estes limites já foram identificados em outros estudos para o uso das tecnologias na escola (AZEVEDO, PIRES \& SILVA, 2007; FAGANELLO-GEMENTE, 2015), sendo um desafio a ser considerado para que os estudos possam de fato promover o uso dos JEMs como ferramenta e conteúdo pedagógico.

\section{Considerações finais}

As pesquisas encontradas indicam que os JEMs podem ser usados pelo campo da Educação Física como um aliado ainda pouco explorado enquanto instrumento de intervenção e como conteúdo pedagógico.

Nos aspectos relacionados com a saúde os artigos apontam o uso dos JEMs como um instrumento de intervenção promissor na reabilitação de pessoas com doenças ou deficiências que apresentam déficit de equilíbrio e no desenvolvimento da agilidade de pacientes com parkinson. Esta positividade encontrada nos possibilita indicar que sejam realizados outros estudos com populações com outras patologias e deficiências, com a sugestão de um número maior de pacientes sendo avaliados, em especial, para averiguar ganhos já apontados, como: amplitude de movimento, coordenação motora fina e melhoras nas atividades diárias.

Além de instrumento de reabilitação os JEMs aparecem nas pesquisas como importantes experiências de promoção da saúde, já que revelam um potencial para a mudança do comportamento sedentário, agindo ainda como incentivo para hábitos alimentares saudáveis. No entanto, as pesquisas de Rodrigues e colaboradores (2013) e de Finco e Fraga (2012), demonstram indiretamente a existência de uma lacuna investigativa sobre os sentidos atribuídos por usuários de JEMs de diferentes grupos etários e sociais. Sentidos que estão relacionados, segundo Bourdieu (2009), com a posição que ocupam estes usuários de JEMs na sociedade. Sobre este aspecto, cabe destacar que os estudos de recepção indicam que os sentidos atribuídos a estas experiências não são homogêneos (MARTÍN-BARBERO, 1997; GÓMEZ, 2005).

A fruição dos usuários com os JEMs não foi considerada nos artigos analisados, ainda que algumas pesquisas identificaram como resultados secundários que a ludicidade era agente motivador da experiência. A relação social, que é um dos destaques dado pelo mercado de JEMs, também não é estudada pelas pesquisas encontradas. Os JEMs enquanto uma prática social desenvolvida com fins de entretenimento carece ter estas duas dimensões, interação social e fruição, investigadas, principalmente, enquanto conteúdo pedagógico. Nesta perspectiva, apesar das pesquisas apontarem o uso dos JEMs na escola, mais especificamente na Educação Física, como ferramenta e conteúdo pedagógico, identifica-se ainda algumas lacunas para pensar suas potencialidades educativas.

Para finalizar, verificou-se que não há estudos sobre a jogabilidade e agência. Jogabilidade é entendida como a capacidade que um jogo tem de proporcionar prazer para quem joga durante um período de tempo prolongado (KÜCKLICH, 2004) e agência como "a capacidade gratificante de realizar ações significativas e ver os resultados de nossas decisões e escolhas" (MURRAY, 2003, p.127). Estudos deste tipo são justificáveis porque possibilitam avaliar com maior propriedade os JEMs como conteúdo e como instrumento de intervenção. Enquanto conteúdo no intuito de saber o que aproxima e o que distancia a experiência com os JEMs das práticas corporais; e enquanto instrumento de intervenção para ter conhecimento do 
seu potencial de aderência e ação na reabilitação, na promoção da saúde e na prática pedagógica.

\title{
MOVEMENT ELECTRONIC GAMES AND PHYSICAL EDUCATION: A SYSTEMATIC REVIEW
}

\begin{abstract}
The purpose of this research is to conduct a systematic review of scientific papers produced on Movement Electronic Games, pointing their uses to the field of Physical Education. The surveys have indicated the use of Motion Electronic Games as an intervention instrument in the field of rehabilitation, health promotion and teaching practice. It appears also as pedagogical content in physical education. The studies do not point the meanings attributed by users other than those related to health.
\end{abstract}

Key words: Video Games. Physical Education. Education. Health.

\section{JUEGOS ELECTRÓNICOS DE MOVIMIENTO Y EDUCACIÓN FÍSICA: UNA REVISIÓN SISTEMÁTICA}

Resumen: La meta de esta pesquisa es realizar una revisión sistemática de los artículos científicos producidos sobre Juegos Electrónicos de Movimiento, apuntando sus usos para el campo de la Educación Física. Las pesquisas encontradas indican el uso de los Juegos Electrónicos de Movimiento como herramienta de intervencíon en el campo de la reabilitacíon, de la promoción de la salud e da la práctica pedagógica. Aparece también como contenido pedagógico en la Educación Física Escolar. Los estudos realizados no apuntan los sentidos atribuidos por los usuários para mas allá de aquellos relacionados a la salud.

Palabras claves: Juegos de Vídeo. Educación Física. Educación. Salud.

\section{Referências}

AMARAL, S. C. F.; PAULA, G. N. A nova forma de pensar o jogo, seus valores e suas possibilidades. In: Pensar a Prática, Goiânia, v.10, n. 2, p. 323-336, Goiânia, jul./dez. 2007.

ARMANDO, J. Lo que podemos aprender de los videojuegos sobre la enseñanza y los materiales educativos. In: Revista Latinoamericana de Tecnología Educativa RELATEC, v.9, n.1, p.29-41, 2010.

AZEVEDO, V. A.; PIRES, G. L.; SILVA, A. P. S. Jogos eletrônicos e suas possibilidades educativas. In: Motrivivência, Santa Catarina, ano 19, n. 28, p. 90-100, jul. 2007.

BARACHO A. F. O.; GRIPP F. J.; LIMA M. R. Os exergames e a educação física escolar na cultura digital. In: Rev. Bras. Ciênc. Esporte, Florianópolis, v. 34, n. 1, p. 111-126, jan./mar. 2012.

BISCAIA, R.; MARTINS, R.; ALVES, S. Aplicabilidade do jogo MoveFitness como ferramenta de treino aeróbio: Uma comparação com diretrizes atuais que definem os vários níveis de intensidade de treino aeróbio. In: Salutis scientia. v.6, p. 32-9, nov. 2014.

BOURDIEU, P. O Senso Prático. Petrópolis/RJ: Vozes, 2009. 
COUTINHO, A. J. A. P.; MATOS, T. P. G. Avaliação dos efeitos de um protocolo com a plataforma Nintendo Wii ${ }^{\circledR}$ nas dimensões cognitivas e físicas (equilíbrio e coordenação) em idosos. In: Rev. Psicol. v. 2, n. 1, p .55-62, 2014.

FAGANELlO GEMENTE, F. R. Atletismo na Educação Física Escolar: a elaboração colaborativa do software ATLETIC. P. 215f. Tese (Doutorado em Desenvolvimento Humano e Tecnologias) - Programa de Pós-Graduação em Desenvolvimento Humano e Tecnologias, UNESP, Rio Claro, 2015.

FANTIN, M. Mídia-educação: conceitos, experiências diálogos Brasil-Itália. Cidade Futura: Florianópolis, 2006.

FERES NETO, A.; JUBÉ, C. N. Redes Sociais, Jogos Eletrônicos e Lazer. In: MELO, V. A.; SCHWARTZ, G. M; FERES NETO, A. (Orgs.) Lazer e Tecnologia. Ijuí: Ed. Unijuí, 2012.

FINCO, M. D.; FRAGA, A. B. Rompendo fronteiras na Educação Física através dos videogames com interação corporal. In: Rev. Motriz : Revista de Educação Física (Online), Rio Claro, v. 18, p. 533-541, 2012.

FINCO, M. D.; FRAGA, A. B. Corpo joystick: cinema, videogames e estilo de vida ativo. In: Rev. Licere (Online), Belo Horizonte, v.16, n.3, set/2013.

FRADE, M. C. M. et al. Equilíbrio dos deficientes visuais antes e após gameterapia. In: Rev. Edu. Espec. v. 27, n. 50, p. 751-764, 2014.

FRANCO, R. C. et al. A realidade virtual como ferramenta para melhorar o equilíbrio dinâmico e a velocidade da marcha na doença de Charcot Marie Tooth: Relato de Caso. In: Rev. Ter. Man. v. 11, n. 53, p. 410-414, 2013.

GRANDE, A. A. B.; GALVÃO, F. R. D.; GONDIM, L. C. A. Reabilitação virtual através do videogame: relato de caso no tratamento de um paciente com lesão alta dos nervos mediano e ulnar. In: Acta Fisiatr. v. 18, n. 3,p. 157-162, 2011.

GÓMEZ, G. O. "O telespectador frente à televisão: uma exploração do processo de recepção televisiva”. Communicare. São Paulo, v. 5, n. 1, 2005, p. 27-42.

HARDY, L. R.; HARDEL.J. S.; BELL, R. A. Overweight in children: definitions measurements confuning factores and heath consequences. In: Journal of Pediatric Nuring. P. 376-384; 19(6), 2104.

KÜCKLICH, J. Play and Playability as Key Concepts in New Media Studies. In: STem Centre, Dublin City University, Dublin, 2004.

MARTIN-BARBERO, J.. Dos Meios às Mediações: comunicação, cultura e hegemonia. Rio de Janeiro: Ed. UFRJ, 1997.

MEDEIROS, C. G. et al. Os benefícios da inclusão da realidade virtual no tratamento fisioterapêutico de um paciente com traumatismo raquimedular (trm): um estudo de caso. In: Caderno de Ciências Biológicas e da Saúde, Boa Vista. (1), 2013. 
MURRAY, J. H. Hamlet no Holodeck: o futuro da narrativa no ciberespaço. São Paulo: Itaú Cultural - UNESP, 2003.

MUSSATO, R.; BRANDALIZE, D.; BRANDALIZE. M.; Nintendo Wii® e seu efeito no equilíbrio e capacidade funcional de idosos saudáveis. In: Rev. Bras. Ci. e Mov. v. 20, n. 2, p. $68-75,2012$.

PAVÃO, S. L. et al. Impacto de intervenção baseada em realidade virtual sobre o desempenho motor e equilíbrio de uma criança com paralisia cerebral: estudo de caso. In: Rev. Paul. Pediatr. v. 32, n. 4, p. 389-394, 2014.

PERIARD, L.V. L.; ELIAS FILHO, J.; AUGUSTO-SILVA, P. Análise da utilização do Nintendo Wii ${ }^{\circledR}$ na melhora funcional do membro superior de um paciente com sequela de AVE. In: Persp. Online: Biol. \& Saúde. v. 8, n. 3, p. 54-63, 2013.

PERRIER-MELO, R. J. et al. Respostas agudas da frequência cardíaca e da pressão arterial em uma sessão de jogos de vídeo game ativos em adultos saudáveis: um estudo piloto. In: Rev. Ter Ocup Univ São Paulo. v. 243, p. 259-66, set./dez., 2013.

RESENDE, M. S.; SILVA, A. P. S. Jogar off e jogar online: compreensões acerca da interação nos jogos eletrônicos. In: Revista Contemporânea (UERJ. Online), v. 10, p. s/p, 2012.

RIVOltella, P. C. Media Education, Fondamenti didattici e prospettive di ricerca. Brescia: La Scuola, 2005.

RODRIGUES, M. A. À sombra das chuteiras virtuais: futebol e lazer nas quatro linhas do jogo eletrônico. p. 96f. Dissertação (Mestrado em Lazer) - Programa Interdisciplinar de Pós-graduação em Lazer, UFMG, Belo Horizonte, 2011.

RODRIGUES, N. H. et al. Experiência subjetiva de idosas durante exercício em ambiente virtual. In: Motriz, Rio Claro, v.19 n.3, Suplemento, p.S68-S75, jul/set. 2013.

SANTO, R. C. E. et al. Dispêndio energético durante a prática de Exergames: um estudo com crianças da região sul do Brasil. In: Rev. Bras. Ativ. Fis. e Saúde. Pelotas/RS v. 19 n. 6, p. 755-756, nov. 2014.

SCHIAVINATO, A. M. et al. Influência do Wii Fit no equilíbrio de paciente com disfunção cerebelar: estudo de caso. In: Journal of Health Sciences Institute. v. 28, n. 1, p. 50-52, 2009.

SILVA, A. P. S. Os Jogos Eletrônicos de Movimento e as Práticas Corporais na percepção de jovens. p. 274f. Tese (Doutorado em Educação Física) - Programa de PósGraduação em Educação Física, UFSC, Florianópolis, 2012.

SILVA, F. C. T.; BRACCIALLI, L. M. P. Programa de Atividade Física Adaptada com Uso de Vídeo Game: o que Pensam os Alunos?. In: Revista da Sobama, Marília, v. 15 n.2, p. 0914, jul./dez., 2014. 
SILVA, G. O. et al. Efeito imediato de intervenções fisioterapêuticas no equilíbrio e na agilidade de parkinsonianos. In: Rev. Ter. Man. v.9, n. 45, p. 569-576, 2011.

SILVA, M. P. et al. Comportamento sedentário relacionado ao sobrepeso e obesidade em crianças e adolescentes. In: Pensar a Prática. v. 13, n. 12, p. 1-15, Goiânia, 2010.

SILVA, R. R.; IWABE-MARCHESE, C.; Uso da realidade virtual na reabilitação motora de uma criança com Paralisia Cerebral Atáxica: estudo de caso. In: Fisioter Pesq. v. 22, n. 1, p. 97-102, 2015.

SOUZA, R. A. et al. Respostas cardiovasculares agudas em ambiente virtualmente simulado pelo Nintendo Wii. In: Rev. Bras. Cineantropom. Desempenho Hum. v. 15, n. 1, p. 60-70, 2013.

SPOSITO, L. A. C. et al. Experiência de treinamento com Nintendo Wii sobre a funcionalidade, equilíbrio e qualidade de vida de idosas. In: Rev. Bras. Motriz, Rio Claro. v.19, n. 2,p. 532-540, 2013.

TOLOCKA, R. E.; COELHO,V.A.C.; SAI,.TG.; DE MARCO, A. CESAR,M.C.; SANTOS,D.C.C. Perfil de crescimento e estado nutricional em crianças e creches e préescolares do município de Piracicaba. Revista de Educação Física, Maringá, v. 19, n. 3, p. 343-351, 2008.

TREML, C. J. et al. O uso da plataforma Balance Board como recurso fisioterápico em idosos. In: Rev. Bras. Geriatr. Gerontol. Rio de Janeiro, v. 16, n. 4, p. 759-768, 2013.

VAGHETTI, C. A. O., et al. Exergames: um desafio à educação física na era da tecnologia. In: Rev. Educação \& Tecnologia. v. 12, p. 1-15, 2012.

VAGHETTI, C. A. O.; MUSTARO, P. N.; BOTELHO, S. S. C. Exergames no ciberespaço: uma possibilidade para a educação física. In: Tecnologia Educacional, v. 40, p. 32, 2011.

VIEIRA, K. L. et al. Características comportamentais de escolares e sua percepção sobre a utilização dos exergames nas aulas de educação física. In: Cinergis. v. 15 n. 2, p. 65-69, abr./jun., 2014.

ZEIGELBOIM, B. S. et al. Reabilitação vestibular com realidade virtual na ataxia espinocerebelar. In: ACR. v.18, n.2, p.143-147, 2013.

Recebido em: 21/12/2015

Revisado em: 12/05/2016

Aprovado em: 16/05/2016

Endereço para correspondência:

letmobile@gmail.com

Letícia de Castro Silva Monteiro

Univesidade Federal de Goiás - Faculdade de Educação Física e Dança

Avenida Esperança, s/n

Campus Samambaia

74690-900 - Goiânia, GO - Brasil 